\title{
Prediction of acute kidney injury in cirrhotic patients: a new score combining renal, liver and inflammatory markers
}

This article was published in the following Dove Press journal: International Journal of Nephrology and Renovascular Disease

\author{
Joana Gameiro' \\ José Agapito Fonseca' \\ Joana Monteiro Dias' \\ Maria João Melo' \\ Sofia Jorge' \\ José Velosa ${ }^{2}$ \\ José António Lopes' \\ 'Division of Nephrology and Renal \\ Transplantation, Department of \\ Medicine, Centro Hospitalar Lisboa \\ Norte, Lisboa, Portugal; ${ }^{2}$ Department \\ of Gastroenterology and Hepatology, \\ Centro Hospitalar Lisboa Norte, \\ Lisboa, Portugal
}

\begin{abstract}
Introduction: Acute kidney injury (AKI) is common in hospitalized patients with cirrhosis and is associated with poor prognosis. A risk prediction score combining values easily measured at admission could be valuable to stratify patients for prevention, monitoring and early intervention, ultimately improving patient care and outcomes. The aim of this study was to develop a risk score for AKI in a cohort of cirrhotic patients.
\end{abstract}

Patients and methods: We cross-examined the data from a retrospective analysis of 186 patients with cirrhosis admitted to the Gastroenterology and Hepatology Service of Centro Hospitalar Lisboa Norte from January 2003 to December 2005. AKI was defined as an increase in serum creatinine ( $\mathrm{SCr}) \geq 0.3 \mathrm{mg} / \mathrm{dL}$ within 48 hours or a percentage increase in $\mathrm{SCr} \geq 50 \%$ from baseline. Neutrophil-to-lymphocyte ratio (NLR) was used as a marker for inflammation. A receiver operating characteristic (ROC) curve was produced to assess the discriminative ability of the variables. Cutoff values were defined as those with highest validity. The final AKI risk score model was assessed using the ROC curve.

Results: A total of 52 patients (28\%) developed AKI. Higher baseline $\mathrm{SCr}(p<0.001)$, more severe liver disease as evaluated by the modified Model of End-stage Liver Disease (MELD)-Na score $(p<0.001)$ and higher NLR $(p=0.028)$ were independently associated with AKI. The area under the ROC (AUROC) curve for the prediction of AKI was 0.791 (95\% CI 0.726-0.847) for SCr, 0.771 (95\% CI 0.704-0.829) for modified MELD-Na and 0.757 (95\% CI 0.689-0.817) for NLR. Cutoff values with the highest validity for predicting AKI were determined and defined as 0.9 for the SCr, 21.7 for the modified MELD-Na and 6 for the NLR. The risk score was created allowing 3 points if the $\mathrm{SCr}$ is higher than $0.9,1$ point if the modified MELD-Na is higher than 21.7 and 1 point if the NLR is higher than 6 . The AUROC curve of the risk prediction score for AKI was 0.861 . A risk score of $\geq 2$ points predicts AKI in cirrhotic patients with a sensitivity of $88.5 \%$ and specificity of $72.4 \%$.

Conclusion: A new score combining SCr, MELD-Na and NLR demonstrated a strong discriminative ability to predict AKI in cirrhotic patients.

Keywords: acute kidney injury, cirrhosis, risk score

\section{Introduction}

Acute kidney injury (AKI) affects between $20 \%$ and $50 \%$ of hospitalized patients with cirrhosis ${ }^{1-3}$ and is associated with poor prognosis with mortality rates reaching as high as $90 \% .^{4}$

Considering the limitations of the traditional markers of renal function in cirrhotic patients, serum creatinine ( $\mathrm{SCr}$ ) and urinary output (UO), studies have focused on new biomarkers to increase diagnostic accuracy, but these are still far from clinical use. ${ }^{5,6}$
Division of Nephrology and Rena Transplantation, Department of Medicine, Centro Hospitalar Lisboa Norte, EPE, Avenue Prof. Egas Moniz, 1649-035 Lisbon, Portugal Email joana.estrelagameiro@gmail.com 
The severity of liver disease has been associated with $\mathrm{AKI}$ and is an important risk factor. ${ }^{7}$ In addition, the role of inflammation in AKI has had increasing recognition and has been reported as a prognostic factor in cirrhotic patients. ${ }^{7}$

Therefore, future biomarkers would have to consider markers of renal and liver function and inflammation markers. The role of the neutrophil-to-lymphocyte ratio (NLR) in predicting AKI has been reported, but it has not been studied in cirrhotic patients. ${ }^{8,9}$

A score combining values easily measured at admission to predict the risk of AKI could be a valuable tool to stratify patients for prevention, monitoring and early intervention and ultimately to improve patient care and outcomes.

The aim of this study was to develop a risk prediction score for AKI in cirrhotic patients, which combined renal and liver dysfunction markers as well as an inflammation marker.

\section{Patients and methods Study design}

To investigate a potential risk score, we cross-examined the data from retrospective analysis of 186 patients with cirrhosis who were admitted to the Gastroenterology and Hepatology Service of Centro Hospitalar Lisboa Norte from January 2003 to December $2005 .{ }^{10}$ Centro Hospitalar Lisboa Norte, Entidade Pública Empresarial (EPE), is an academic and referral center serving a population of $3,000,000$ inhabitants. The study was approved by the ethical committee at the Centro Hospitalar Lisboa Norte, EPE, in agreement with institutional guidelines. Informed consent was waived by the ethical committee due to the retrospective and noninterventional nature of the study.

\section{Participants}

All patients aged 18 years or older who were admitted to the Gastroenterology and Hepatology Service of Centro Hospitalar Lisboa Norte from January 2003 to December 2005 were eligible for this study. All patients' data accessed were de-identified to preserve anonymity.

Exclusion criteria included the following: chronic kidney disease patients already on renal replacement therapy; patients who underwent renal replacement therapy the week before admission and patients who had less than two determinations of SCr during hospital stay.

\section{Variables}

All variables were collected from electronic and handwritten patient clinical records. All scores and formulas were calculated based on clinical data.

The analyzed variables included demographic characteristics (age, gender and ethnicity), etiology of liver disease, comorbidities, reason for admission, laboratory data including baseline $\mathrm{SCr}$ and, at admission, hemoglobin, neutrophils, leukocytes, international normalized ratio (INR), bilirubin, sodium and albumin, need for vasopressors and mechanical ventilation, AKI, mortality and length of hospital stay.

Regarding clinical characteristics, the comorbidities registered were diabetes mellitus (diagnosed according to the American Diabetes Association criteria) ${ }^{11}$ hypertension (diagnosed according to the seventh report of the Joint National Committee), ${ }^{12}$ cardiovascular disease (including chronic heart failure, cardiac ischemic disease and history of transient ischemic attack or stroke) and malignancy. The severity of liver disease was assessed with the model for end-stage liver disease incorporating sodium (Model of End-stage Liver Disease [MELD]-Na) and Child-Pugh scores. ${ }^{13-15}$ A modified MELD-Na score not including $\mathrm{SCr}$ was calculated to exclude variable collinearity.

$$
\begin{gathered}
\text { Modified MELD }-\mathrm{Na}=\text { Modified MELD }-\mathrm{Na}- \\
0.025^{*} \text { Modified MELD }(140-\mathrm{Na})+140 \\
\text { Modified MELD }=0.378 \times \ln (\text { bilirubin })+ \\
1.120 \times \ln (\mathrm{INR})+0.643
\end{gathered}
$$

AKI was defined as an increase in $\mathrm{SCr} \geq 0.3 \mathrm{mg} / \mathrm{dL}$ within 48 hours or percentage increase in $\mathrm{SCr} \geq 50 \%$ from baseline. $\mathrm{SCr}$ at admission was considered baseline $\mathrm{SCr}$.

Cirrhosis was diagnosed by liver biopsy or a combination of biochemical, radiological and endoscopic findings when liver biopsy was not available.

NLR was determined using neutrophil and lymphocyte counts at admission. NLR was calculated as follows: neutrophil count/lymphocyte count.

\section{Statistical analyses}

Continuous variables were presented as mean \pm SD and categorical variables as the total number and percentage of cases for each category. After grouping participants according to the development of AKI, the variables of both groups were compared using Student's $t$-test for normally distributed continuous variables, Mann-Whitney $U$-test for non-normally distributed continuous variables and chi-square test for categorical variables.

Only variables that significantly differed between AKI and non-AKI groups were used in the univariate and multivariate analysis using the logistic regression method. Data were expressed as ORs with $95 \%$ CI. No sensitivity analyses were carried out.

A receiver operating characteristic (ROC) curve was produced to assess the discriminative ability of the variables for 
AKI. Cutoff values were defined as those with highest validity for predicting AKI. The coefficients produced for each variable in the multivariate model were rounded to the nearest numeral to develop the risk score. By adding the variables together, the total score can range from a minimum of 0 to a maximum of 5 points. The final AKI risk score model was assessed using the area under the ROC (AUROC) curve and Hosmer-Lemeshow goodness-of-fit test.

Statistical significance was defined at a $p$-value of $<0.05$. Analyses were performed with the statistical software package SPSS 21.0 (IBM Corporation, Armonk, NY, USA) for Windows.

\section{Results}

Demographic patient variables and outcomes including comparisons between the AKI and non-AKI groups are described in Table 1. We registered no missing data.

In our cohort, $28 \%$ of patients $(n=52)$ developed AKI (42.3\% stage $1,34.6 \%$ stage 2 , and $23.1 \%$ stage 3 ). A total of 12 patients with AKI (23.1\%) underwent renal replacement treatment. Median time to the occurrence of AKI following admission was 2 days (1-24 days). Median SCr on the day of AKI diagnosis was $2.1 \pm 1.2 \mathrm{mg} / \mathrm{dL}$. AKI patients were more likely to require mechanical ventilation $(p=0.028)$ and vasopressors $(p=0.001)$. Comorbidity, etiology of liver disease and reason for admission were not associated with AKI.

A higher baseline $\mathrm{SCr}(1.8 \pm 1.2$ vs $0.9 \pm 0.4, p<0.001$; unadjusted OR 4.6 [95\% CI 2.5-8.6], $p<0.001$; adjusted OR 3.4 [95\% CI 1.8-6.2], $p<0.001$ ), more severe liver disease as evaluated by the modified MELD-Na score (22.8 \pm 7.4 vs 15.7 $\pm 6.3, p<0.001$; unadjusted OR 1.2 [95\% CI 1.1-1.3], $p<0.001$; adjusted OR 1.15 [95\% CI 1.1-1.2], $p<0.001)$ and higher NLR (13.9 \pm 16.5 vs $5.5 \pm 4.0, p<0.001$; unadjusted OR 1.2 [95\% CI 1.1-1.3], $p<0.001$; adjusted OR 1.1 [95\% CI 1.0-1.1], $p=0.028$ ) were independently associated with AKI (Table 2).

The AUROC curve for the prediction of AKI was 0.791 (95\% CI 0.726-0.847) for SCr, 0.771 (95\% CI 0.704-0.829) for modified MELD-Na and 0.757 (95\% CI 0.689-0.817) for NLR. Cutoff values with the highest validity for predicting AKI were determined and defined as 0.9 for the $\mathrm{SCr}, 21.7$ for the modified MELD-Na and 6 for the NLR (Table 3).

The risk score was created allowing 3 points if the $\mathrm{SCr}$ is higher than 0.9, 1 point if the modified MELD-Na is higher than 21.7 and 1 point if the NLR is higher than 6 (Table 4).

The AUROC curve of the risk prediction score for AKI was 0.861 (95\% CI 0.803-0.908; Figure 1). The optimal cutoff for the diagnosis of AKI was assessed to be $\geq 2$ points,
Table I Characteristics of patients with and without AKI

\begin{tabular}{|c|c|c|c|}
\hline Variables & $\begin{array}{l}\text { AKI } \\
(n=52)\end{array}$ & $\begin{array}{l}\text { No AKI } \\
(n=134)\end{array}$ & $p$-value \\
\hline \multicolumn{4}{|l|}{ At admission } \\
\hline Age (years) & $57.9 \pm 10.3$ & $55.3 \pm 12.8$ & 0.220 \\
\hline Male, n (\%) & $45(86.5)$ & $93(73.1)$ & 0.052 \\
\hline Caucasian, n (\%) & $46(88.5)$ & I I 7 (87.3) & 0.831 \\
\hline \multicolumn{4}{|l|}{ Comorbidity, n (\%) } \\
\hline Diabetes mellitus & $12(23.1)$ & $38(28.4)$ & 0.729 \\
\hline Hypertension & $19(36.5)$ & $37(27.6)$ & 0.096 \\
\hline Cardiovascular disease & $12(23.1)$ & $24(17.9)$ & 0.250 \\
\hline Cancer & $5(9.6)$ & $9(6.7)$ & 0.379 \\
\hline \multicolumn{4}{|l|}{ Etiology of liver disease, n (\%) } \\
\hline Alcohol consumption & $31(59.6)$ & $84(62.7)$ & 0.647 \\
\hline \multicolumn{4}{|l|}{ Alcohol consumption plus } \\
\hline $\mathrm{HCV}$ infection & $5(9.6)$ & $24(17.9)$ & 0.249 \\
\hline \multicolumn{4}{|l|}{ Alcohol consumption plus } \\
\hline HBV infection & I (I.9) & $4(3)$ & 0.763 \\
\hline HCV infection & $2(3.8)$ & II (8.2) & 0.295 \\
\hline HBV infection & $4(7.7)$ & $3(2.2)$ & 0.054 \\
\hline Others & $6(I 1.5)$ & $9(6.7)$ & 0.506 \\
\hline \multicolumn{4}{|l|}{ Reason for admission, $\mathrm{n}(\%)$} \\
\hline Gastrointestinal bleeding & $26(50)$ & $86(64.2)$ & 0.076 \\
\hline Sepsis & $17(32.7)$ & $26(19.4)$ & 0.054 \\
\hline Liver failure & $2(3.8)$ & $6(4.5)$ & 0.849 \\
\hline Others & $5(9.6)$ & $16(11.9)$ & 0.125 \\
\hline \multicolumn{4}{|l|}{ Laboratory at admission } \\
\hline Hemoglobin $(\mathrm{g} / \mathrm{L})$ & $9.2 \pm 2.3$ & $9.5 \pm 1.9$ & 0.393 \\
\hline $\mathrm{SCr}$ & $1.8 \pm 1.2$ & $0.9 \pm 0.4$ & $<0.001$ \\
\hline NLR & $13.9 \pm 16.5$ & $5.5 \pm 4.0$ & $<0.001$ \\
\hline Modified MELD-Na & $22.8 \pm 7.4$ & $15.7 \pm 6.3$ & $<0.001$ \\
\hline Child-Pugh & $34.6 \pm 5.5$ & $33.1 \pm 7.8$ & 0.189 \\
\hline \multicolumn{4}{|l|}{ During hospitalization } \\
\hline MV requirement, n (\%) & $13(25)$ & $16(11.9)$ & 0.028 \\
\hline Vasopressors requirement, $\mathrm{n}(\%)$ & $18(34.6)$ & $17(12.7)$ & 0.001 \\
\hline Length of hospital stay (days) & $(8.8 \pm 12.9)$ & $12.1 \pm 12.4$ & 0.002 \\
\hline In-hospital mortality, n (\%) & $25(48)$ & $9(6.7)$ & $<0.0001$ \\
\hline
\end{tabular}

Abbreviations: $A K I$, acute kidney injury; $\mathrm{HBV}$, hepatitis $B$ virus; $\mathrm{HCV}$, hepatitis $\mathrm{C}$ virus; MELD, Model of End-stage Liver Disease; MV, mechanical ventilation; NLR, neutrophil-to-lymphocyte ratio; $\mathrm{SCr}$, serum creatinine.

which had a sensitivity of $88.5 \%$ and specificity of $72.4 \%$. This cutoff identified $44.6 \%(n=83)$ of the population at risk for developing AKI, of whom 55.4\% will develop AKI $(n=46)$. In contrast, $94.2 \%$ of patients with risk score of $<2$ will not develop AKI $(p<0.001)$.

\section{Discussion}

The high incidence of AKI in cirrhotic patients and its association with worse outcomes have raised interest in developing biomarkers to prevent $\mathrm{AKI}$ in patients at risk and improve diagnostic criteria.

In this study, we developed a low-cost and easily calculated risk score for the prediction of AKI in patients with cirrhosis. This is a low-cost risk score, easily calculated from 
Table 2 Univariate and multivariate analysis of factors predictive of AKI

\begin{tabular}{|c|c|c|c|c|}
\hline \multirow[t]{2}{*}{ Variables } & \multicolumn{4}{|l|}{ AKI } \\
\hline & Unadjusted OR (95\% Cl) & $p$-value & Adjusted OR (95\% Cl) & $p$-value \\
\hline \multicolumn{5}{|c|}{ Demographic characteristics } \\
\hline Age & $1.02(0.99-1.05)$ & 0.241 & & \\
\hline Male & $0.42(0.18-1.02)$ & 0.057 & & \\
\hline Caucasian & I.II (0.4I-3.0) & 0.831 & & \\
\hline \multicolumn{5}{|l|}{ Parameters at admission } \\
\hline $\mathrm{Hb}(\mathrm{g} / \mathrm{L})$ & $1.0(0.9-1.3)$ & 0.545 & & \\
\hline $\mathrm{SCr}$ & $4.6(2.5-8.6)$ & $<0.001$ & $3.4(1.8-6.2)$ & $<0.001$ \\
\hline NLR & $1.2(1.1-1.3)$ & $<0.001$ & I.I (I.0-I.I8) & 0.028 \\
\hline Modified MELD-Na & $1.2(1.1-1.3)$ & $<0.001$ & $1.15(1 . I-1.2)$ & $<0.001$ \\
\hline Child-Pugh & $1.03(0.98-1.09)$ & 0.191 & & \\
\hline
\end{tabular}

Abbreviations: AKI, acute kidney injury; Hb, hemoglobin; MELD, Model of End-stage Liver Disease; NLR, neutrophil-to-lymphocyte ratio; SCr, serum creatinine.

Table 3 Univariate and multivariate analysis of categorical variables

\begin{tabular}{lllll}
\hline Variables & AKI & & & \\
\cline { 2 - 5 } & Unadjusted OR (95\% Cl) & p-value & Adjusted OR (95\% Cl) & p-value \\
\hline SCr $>0.9$ & $10.2(4.8-21.7)$ & $<0.001$ & $6.9(2.9-15.9)$ & $<0.001$ \\
NLR $>6$ & $5.9(2.9-11.9)$ & $<0.001$ & $2.4(1.0-5.8)$ & 0.041 \\
Modified MELD-Na>2I.7 & $11.7(5.6-24.8)$ & $<0.001$ & $6.9(2.9-16.3)$ & $<0.001$ \\
\hline
\end{tabular}

Abbreviations: AKI, acute kidney injury; MELD, Model of End-stage Liver Disease; NLR, neutrophil-to-lymphocyte ratio; SCr, serum creatinine.

Table 4 Risk prediction score

\begin{tabular}{ll}
\hline Risk factor & Points \\
\hline $\mathrm{SCr}>0.9$ & 3 \\
$\mathrm{NLR}>6$ & $\mathrm{I}$ \\
MELD-Na $>21.7$ & $\mathrm{I}$ \\
Total & 5 \\
Score to predict AKI & $\geq 2$ \\
\hline
\end{tabular}

Abbreviations: AKI, acute kidney injury; MELD, Model of End-stage Liver Disease; NLR, neutrophil-to-lymphocyte ratio; SCr, serum creatinine.

a complete blood count and biochemistry panel collected at hospital admission, which can reliably predict AKI with AUROC of 0.861 in cirrhotic patients at admission.

The variables used as risk factors for the prediction of $\mathrm{AKI}$ in this score are consistent with the previous literature. ${ }^{6-8}$

Although $\mathrm{SCr}$ is the most common renal dysfunction biomarker, its limitations in cirrhotic patients are well documented. ${ }^{6,16,17}$ Multiple factors contribute to lower SCr values in this population, namely decreased hepatic creatine synthesis, decreased muscle mass and increased tubular creatinine secretion. ${ }^{6,16,17}$

The incidence of AKI in cirrhotic patients is variable depending on how baseline renal function is assessed. ${ }^{18}$ The recent Kidney Disease Improving Global Outcome (KDIGO) classification combines the AKI definition of the risk, injury, failure, loss of kidney function and end-stage kidney disease (RIFLE) and AKI network (AKIN) classifications and its

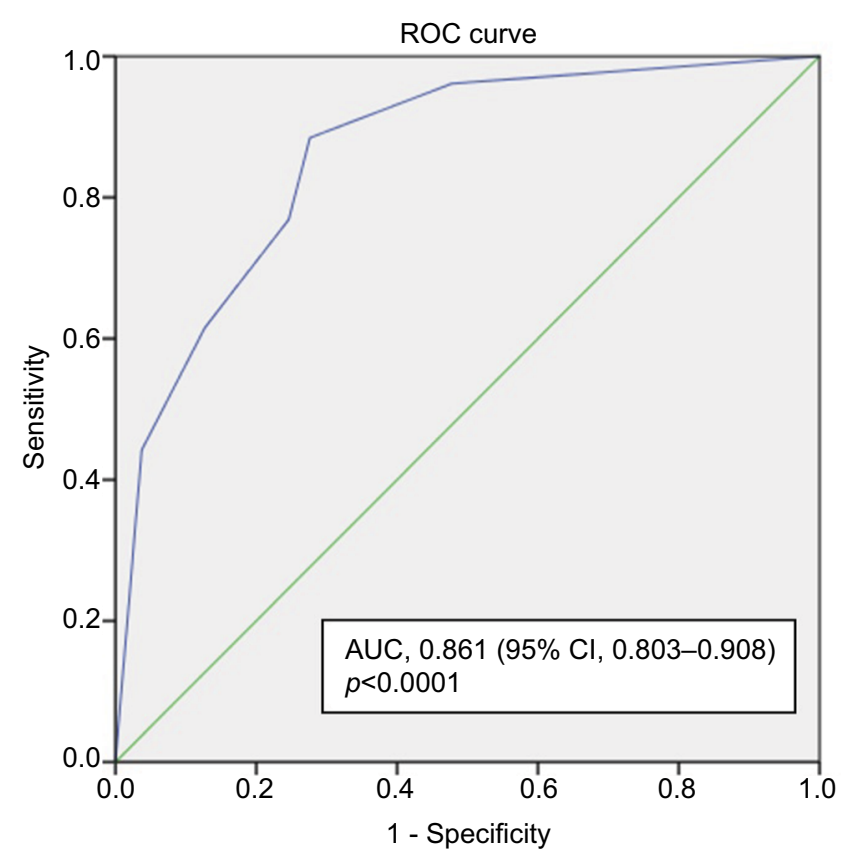

Figure I AUC of the risk model for the prediction of AKI in cirrhotic patients. Abbreviations: AKI, acute kidney injury; AUC, area under the curve; ROC, receiver operating characteristic.

superior diagnostic accuracy and prediction of adverse outcomes has been proven in cirrhotic patients. ${ }^{19-21}$

The KDIGO classification accurately detects minor variations in $\mathrm{SCr}$ values which have been associated with poor prognosis in cirrhotic patients. ${ }^{18,22,23}$ In addition, AKI 
superimposed on previous renal dysfunction confers a higher risk for mortality. ${ }^{18}$ Despite previous SCr thresholds being considered for clinical relevance in cirrhotic patients, ${ }^{24}$ lesser degrees of baseline renal dysfunction can be associated with reduced survival. ${ }^{18,23,25}$ In fact, in our cohort, the baseline $\mathrm{SCr}$ threshold with highest validity for predicting AKI was $0.9 \mathrm{mg} / \mathrm{dL}$.

The MELD score is a validated prognostic marker in a wide range of severity and causes of cirrhosis, which incorporates an assessment of renal function. ${ }^{13}$ Recent variations of this score include incorporating serum sodium (MELD$\mathrm{Na}$ ), which is a significant predictor of early mortality and an indirect marker of ascites. ${ }^{14,26,27}$ In this cohort, higher MELD and MELD-Na were independently associated with AKI. To incorporate MELD-Na in our score, we separated the $\mathrm{SCr}$ from the MELD-Na calculation to assess the impact of the baseline $\mathrm{SCr}$ as a different independent variable.

Besides, Thabut et $\mathrm{al}^{7}$ first reported that the severity of liver disease and inflammation also had an important prognostic role in patients with AKI and cirrhosis. It is well known that cirrhotic patients have a combination of systemic inflammation and immune deficiency, which has been referred as the cirrhosis-associated immune dysfunction (CAID) syndrome. ${ }^{23}$ The pro-inflammatory status of cirrhotic patients, characterized by persistent activation of circulating immune cells and increased levels of pro-inflammatory cytokines, results in recurrent activation of circulating immune cells from damage-associated molecular patterns (DAMPs) that are released from necrotic liver cells and from pathogenassociated molecular patterns (PAMPs) that are released from the intestinal translocation. ${ }^{23,28}$ The increased levels of proinflammatory cytokines induce vascular dysfunction, which worsens systemic vasodilation and renal vasoconstriction. ${ }^{29}$ The disturbance in renal blood flow can cause oxidative stress and tubular damage and lead to AKI. ${ }^{29}$ In addition, inflammation directly affects renal function, through the release of PAMPs and DAMPs, ${ }^{29}$ and plays a central role in the pathogenesis of AKI. ${ }^{30,31}$ In fact, cellular injury and its molecular products are considered important activators of inflammation after AKI. ${ }^{31,32}$ The evidence that norfloxacin administration reduces the severity of renal dysfunction in cirrhotic patients further supports the role of inflammation in AKI. ${ }^{33}$

To incorporate an inflammatory marker in our risk score, we evaluated the NLR which has been recognized as an important marker for inflammation in AKI. ${ }^{8,9,34,35}$ In fact, a higher NLR was independently associated with AKI in our study $(p=0.028)$.
We must consider some potential limitations of our study. First, this was a single-center cohort that limited the generalization of our results. Second, the retrospective design with a relatively small cohort of patients may contribute to overlooking some potential prognostic factors. Third, we did not assess UO in our cohort to define AKI. Finally, we used $\mathrm{SCr}$ at admission as a baseline which can underestimate the incidence of AKI, although glomerular filtration rate-based formulas to assess baseline SCr such as the modification of diet in renal disease (MDRD) tend to overestimate glomerular filtration rate in cirrhosis.

Nevertheless, our study has many important virtues. To our knowledge, it is the first risk score to predict AKI in cirrhotic patients which incorporates renal and liver function assessment as well as inflammation. In addition, the predictive value of our ROC curve is strong, suggesting the potential confirmation of this risk score in other populations.

\section{Conclusion}

We have developed a new easily calculated risk score to predict AKI in cirrhotic patients, which combines markers of renal and liver function and inflammation and deserves further validation in large-scale studies.

\section{Acknowledgments}

No funding was received for this study. The results presented in this study have not been published previously in whole or part.

\section{Disclosure}

The authors report no conflicts of interest in this work.

\section{References}

1. Garcia-Tsao G, Parikh CR, Viola A. Acute kidney injury in cirrhosis Hepatology. 2008;48(6):2064-2077.

2. Tandon P, James MT, Abraldes JG, Karvellas CJ, Ye F, Pannu N. Relevance of new definitions to incidence and prognosis of acute kidney injury in hospitalized patients with cirrhosis: a retrospective populationbased cohort study. Sands JM, ed. PLoS One. 2016;11(8):e0160394.

3. Davenport A, Sheikh MF, Lamb E, Agarwal B, Jalan R. Acute kidney injury in acute-on-chronic liver failure: where does hepatorenal syndrome fit? Kidney Int. 2017;92(5):1058-1070.

4. Fede G, D'Amico G, Arvaniti V, et al. Renal failure and cirrhosis: a systematic review of mortality and prognosis. $J$ Hepatol. 2012;56(4):810-818.

5. Wong F. The evolving concept of acute kidney injury in patients with cirrhosis. Nat Rev Gastroenterol Hepatol. 2015;12(12):711-719.

6. Sherman DS, Fish DN, Teitelbaum I. Assessing renal function in cirrhotic patients: problems and pitfalls. Am J Kidney Dis. 2003;41:269-278.

7. Thabut D, Massard J, Gangloff A, et al. Model for end-stage liver disease score and systemic inflammatory response are major prognostic factors in patients with cirrhosis and acute functional renal failure. Hepatology. 2007;46:1872-1882. 
8. Abu Alfeilat M, Slotki I, Shavit L. Single emergency room measurement of neutrophil/lymphocyte ratio for early detection of acute kidney injury (AKI). Intern Emerg Med. Epub 2017, Jul 29.

9. Yilmaz H, Cakmak M, Inan O, Darcin T, Akcay A. Can neutrophillymphocyte ratio be independent risk factor for predicting acute kidney injury in patients with severe sepsis? Ren Fail. 2015;37(2): 225-229.

10. Lopes JA, Melo MJ, Costa AC, et al. Acute kidney injury and in-hospital mortality in critically ill patients with cirrhosis: a cohort study. Gut. 2012;61(6):955-956.

11. ADA. Standards of medical care in diabetes - 2009. Diabetes Care. 2009;32(supp1 1):S13-S61.

12. Chobanian AV, Bakris GL, Black HR, et al. The Seventh Report of the Joint National Committee on Prevention, Detection, Evaluation, and Treatment of High Blood Pressure: the JNC 7 report. JAMA. 2003;289(19):2560-2572.

13. Kamath PS, Kim WR, Advanced Liver Disease Study Group. The model for end-stage liver disease (MELD). Hepatology. 2007;45(3): 797-805.

14. Kim WR, Biggins SW, Kremers WK, et al. Hyponatremia and mortality among patients on the liver-transplant waiting list. $N$ Engl J Med. 2008;359(10):1018-1026.

15. Pugh RN, Murray-Lyon IM, Dawson JL, et al. Transection of the oesophagus for bleeding oesophageal varices. Br J Surg. 1973;60: 646-649.

16. Angeli $\mathrm{P}$, Ginès $\mathrm{P}$, Wong $\mathrm{F}$, et al. Diagnosis and management of acute kidney injury in patients with cirrhosis: revised consensus recommendations of the International Club of Ascites. $J$ Hepatol. 2015;62(4):968-974.

17. Caregaro L, Menon F, Angeli P, et al. Limitations of serum creatinine level and creatinine clearance as filtration markers in cirrhosis. Arch Intern Med. 1994;154:201-205.

18. Wong F, O'Leary JG, Reddy KR, et al. Acute kidney injury in cirrhosis: baseline serum creatinine predicts patient outcomes. Am J Gastroenterol. 2017;112(7):1103-1110.

19. KDIGO. Clinical practice guideline for acute kidney injury. Kidney Int. 2012;2(1):1-138.

20. Pan HC, Chien YS, Jenq CC, et al. Acute kidney injury classification for critically ill cirrhotic patients: a comparison of the KDIGO, AKIN, and RIFLE classifications. Sci Rep. 2016;6:23022.

21. Biyık M, Ataseven H, Biyık Z, et al. KDIGO (Kidney Disease: Improving Global Outcomes) criteria as a predictor of hospital mortality in cirrhotic patients. Turk J Gastroenterol. 2016;27(2):173-179.
22. de Carvalho JR, Villela-Nogueira CA, Luiz RR, et al. Acute kidney injury network criteria as a predictor of hospital mortality in cirrhotic patients with ascites. J Clin Gastroenterol. 2012;46:e21-e26.

23. Bucsics T, Mandorfer M, Schwabl P, et al. Impact of acute kidney injury on prognosis of patients with liver cirrhosis and ascites: a retrospective cohort study. J Gastroenterol Hepatol. 2015;30(11):1657-1665.

24. Salerno F, Gerbes A, Ginès P, et al. Diagnosis, prevention and treatment of hepatorenal syndrome in cirrhosis. Gut. 2007;56(9):1310-1318.

25. Wong F, O'Leary JG, Reddy KR, et al. A cut-off serum creatinine value of $1.5 \mathrm{mg} / \mathrm{dl}$ for AKI--to be or not to be. J Hepatol. 2015;62(3):741-743.

26. Biggins SW, Kim WR, Terrault NA, et al. Evidence-based incorporation of serum sodium concentration into MELD. Gastroenterology. 2006;130(6):1652-1660.

27. Londono MC, Cardenas A, Guevara M, et al. MELD score and serum sodium in the prediction of survival of patients with cirrhosis awaiting liver transplantation. Gut. 2007;56(9):1283-1290.

28. Dirchwolf M, Ruf AE. Role of systemic inflammation in cirrhosis: from pathogenesis to prognosis. World J Hepatol. 2015;7(16):1974-1981.

29. Giron-Gonzalez JA, Martinez-Sierra C, Rodriguez-Ramos C, et al. Implication of inflammation-related cytokines in the natural history of liver cirrhosis. Liver Int. 2004;24(5):437-445.

30. Gomez H, Ince C, De Backer D, et al. A unified theory of sepsis-induced acute kidney injury: inflammation, microcirculatory dysfunction, bioenergetics, and the tubular cell adaptation to injury. Shock. 2014;41(1):3-11.

31. Akcay A, Nguyen Q, Edelstein CL. Mediators of inflammation in acute kidney injury. Mediators Inflamm. 2009;2009:137072.

32. Bonventre JV, Zuk A. Ischemic acute renal failure: an inflammatory disease? Kidney Int. 2004;66(2):480-485

33. Rabb H, Griffin MD, McKay DB, et al. Inflammation in AKI: current understanding, key questions, and knowledge gaps. J Am Soc Nephrol. 2016;27(2):371-379.

34. Shah N, Dhar D, El Zahraa Mohammed F, et al. Prevention of acute kidney injury in a rodent model of cirrhosis following selective gut decontamination is associated with reduced renal TLR4 expression. J Hepatol. 2012;56(5):1047-1053.

35. Yuan Y, Qiu H, Hu X, et al. Predictive value of inflammatory factors on contrast-induced acute kidney injury in patients who underwent an emergency percutaneous coronary intervention. Clin Cardiol. 2017;40(9):719-725.

36. Kim WH, Park JY, Ok SH, Shin IW, Sohn JT. Association between the neutrophil/lymphocyte ratio and acute kidney injury after cardiovascular surgery: a retrospective observational study. Medicine (Baltimore). 2015;94(43):e1867.

\section{Publish your work in this journal}

The International Journal of Nephrology and Renovascular Disease is an international, peer-reviewed open access journal focusing on the pathophysiology of the kidney and vascular supply. Epidemiology, screening, diagnosis, and treatment interventions are covered as well as basic science, biochemical and immunological studies. The manuscript management system is completely online and includes a very quick and fair peer-review system, which is all easy to use. Visit http://www. dovepress.com/testimonials.php to read real quotes from published authors. 